\title{
Une aide à la coopération interentreprises pour la production à la commande
}

\author{
Emmanuelle Monsarrat * _ Cyril Briand *,** \\ Patrick Esquirol $*$,***
}

\author{
* LAAS - CNRS \\ 7, avenue du colonel roche \\ F-31077 Toulouse cedex 4 \\ \{monsarrat, briand, esquirol\}@laas.fr \\ ** Université Paul Sabatier \\ 118 route de Narbonne \\ F-31062 Toulouse cedex 4 \\ *** Institut National des Sciences Appliquées \\ 135 avenue de rangueil \\ F-31077 Toulouse cedex 4
}

\begin{abstract}
RÉSUMÉ. Cet article propose une méthode d'aide à la coopération interentreprises dans le cadre d'une production à la commande. L'approche retenue assimile le problème d'organisation globale à un processus de décision distribuée dans lequel l'organisation est progressivement construite par un ensemble de coopérations entre des couples d'acteurs du réseau d'entreprises. Nous étudions en particulier les couples d'entreprises donneur d'ordre-fournisseurpour lesquels la coopération concerne les attributs des commandes passées entre eux. Nos objectifs sont de fournir aux décideurs un cadre plus formel qui contractualise la coopération et des outils d'aide à la coopération permettant de les assister dans les diverses phases du processus. ABSTRACT. This paper proposes a method which assists the firms for cooperating in make-toorder production. In the selected approach, the global organization is achieved progressively by a set of cooperations between pairs of actor of the companies network. We study in particular the process of cooperation which concerns the attributes of orders transmitted by the customers to the suppliers. Our objective is to give a more contractual framework to the cooperation between the decision-makers, and to design support tools that assist them during the cooperation process.
\end{abstract}

MOTS-CLÉS : coopération, réseau d'entreprises, aide à la décision, ordonnancement.

KEYWORDS: cooperation, firms network, decision-aid, scheduling.

DN - 8/2004. Coopération et organisation numériques, pages 23 à 36 


\section{Introduction}

Du fait de la diversification de leur production, ou de leur spécialisation relativement à un type de métier, les entreprises sont de plus en plus souvent amenées à collaborer avec un nombre important de partenaires. Elles constituent ainsi un réseau que l'on désigne communément par le nom de chaînes logistiques. Les nouvelles technologies de l'information et de la communication, en augmentant la qualité et la fréquence des échanges entre les partenaires, favorisent l'émergence de réseaux [SAR 02]. En revanche, elles n'apportent pas de solution générale au problème de la cohérence des décisions individuelles vis-à-vis d'une organisation globale. Ainsi, chaque acteur reste libre de remettre en cause à tout moment des décisions collectives, ce qui peut donc induire une instabilité organisationnelle globale.

Pour pallier ce problème, il est nécessaire d'adopter un fonctionnement plus coopératif dans lequel toute décision locale affectant l'organisation de plusieurs acteurs doit faire l'objet d'un processus de décision collectif. Les bénéfices de la coopération sont alors partagés par l'ensemble des acteurs [LEE 97]. Dans le contexte de l'organisation de production, les bénéfices attendus de la coopération résident dans une meilleure tension des flux de production, une meilleure adaptation de la capacité de travail et une meilleure stabilité de l'organisation au sein de la communauté d'intérêts [CAM 02]. D'autre part, la coopération sous-tend une gestion collective du risque dans la mesure où un acteur peut s'appuyer sur ses partenaires pour absorber les perturbations qui le désorganisent.

Cet article est organisé comme suit. Tout d'abord, il rappelle le contexte et les problématiques liées à la coopération interentreprises dans le contexte de la production à la commande. Il décrit ensuite une approche de coopération pour une relation de type donneur d'ordre-fournisseur et définit un support d'aide à la coopération instrumentant cette relation. Pour ce type de coopération, une aide à la décision est proposée.

\section{Contexte de l'étude et hypothèses de travail}

\subsection{Coopération et production à la commande}

La coopération est habituellement définie comme une action collective organisée autour d'un ensemble d'acteurs partageant un but commun. En ce sens, elle est souvent considérée comme un moyen de dépasser les frontières de l'action individuelle. Face à un problème d'organisation et de coordination d'activités réparties, plusieurs situations de coopération peuvent être discernées comme par exemple : la définition des objectifs, la conception des activités, leur répartition, et enfin leur exécution coordonée. Ces situations impliquent trois types d'actions coopératives complémentaires [SPO 00, CAM 00] : la coordination visant à synchroniser les activités individuelles nécessaires à la réalisation d'une activité ou d'une décision globale, la collaboration qui amène plusieurs acteurs à travailler ensemble à l'élaboration d'une activité, et la codécision dans le cas où les acteurs travaillent à l'élaboration d'une décision. 
De nombreux chercheurs se sont intéressés à l'instrumentation de la coopération et notamment à la conception d'outils logiciels [SPO 00]. La coopération étant un processus dynamique, le choix de l'outil de coopération adéquat dépend du cadre temporel, spatial et organisationnel qui caractérise une situation de coopération [BOU 02]. Les outils de coopération sont classiquement différenciés selon qu'ils permettent une coopération synchrone (les acteurs coopèrent de façon simultanée) ou asynchrone, et selon qu'ils imposent aux acteurs d'être physiquement présents au même endroit. La pertinence de ces outils nécessite également d'être évaluée en situation réelle comme suggéré dans [BAK 01].

L'approche proposée dans cet article s'inscrit dans le champ de la coopération pour la production à la commande. La production à la commande correspond à une production en petites et moyennes séries pour laquelle les gammes de fabrication sont prédéfinies. Elle est caractérisée par une demande fortement imprévisible. De ce fait, la création de stocks est évitée, et tout processus de fabrication n'est initié qu'à partir du moment où une commande a été contractée par un donneur d'ordre. Pour ce type de production, l'objectif principal est de tendre les flux de produits, de façon à minimiser le temps s'écoulant entre l'occurrence d'une commande et la date de mise à disposition effective des produits auprès du donneur d'ordre.

Afin de diminuer la complexité de la gestion de production, cette dernière est classiquement décomposée en trois fonctions principales [ART 02]. La fonction planification construit à moyen terme un plan de production qui définit les quantités à produire pour chaque ressource sur un ensemble de périodes élémentaires. Sur la base du plan de production, la fonction ordonnancement définit un plan de fabrication détaillé à court terme précisant les dates de début et de fin de chaque activité. La fonction pilotage exécute en temps réel le plan de fabrication en réagissant aux aléas inhérents à ce type de production (retards, pannes de ressources, ruptures d'approvisionnement...) et en maîtrisant la performance.

Les activités à réaliser étant supposées parfaitement déterminées, la coopération consiste à organiser et synchroniser les activités chez l'ensemble des partenaires. Ces actions concernent deux processus de coopération complémentaires [TRE 98]. Le premier correspond à un processus de coopération vertical, qui reflète la hiérarchie des fonctions de la gestion de production. Le second correspond a un processus de coopération horizontal qui concerne la réalisation distribuée d'une fonction de production particulière.

De nombreux travaux de recherche se sont intéressés à l'instrumentation de ces processus. Certains préconisent une approche centralisée faisant l'hypothèse de l'existence d'une entité qui, ayant une vision globale des caractéristiques des flux sur l'ensemble du processus, superviserait toutes les activités [ART 02]. Parmi les travaux apparentés à ce type d'approche, un exemple de coopération horizontale au niveau planification est présenté dans [BOU 01] où des modèles macroscopiques sont proposés, garantissant une cohérence globale de la planification et de l'allocation de charge dans un contexte intra ou interentreprises. Un exemple de coopération verticale est également proposé dans [GUI 00] concernant le couplage entre une planification glo- 
bale et des ordonnancements locaux. Un inconvénient des approches centralisées est qu'elles requièrent une totale transparence des acteurs, ceux-ci devant communiquer à l'entité superviseur les caractéristiques de leur production. Cette hypothèse semble peu acceptable dans un contexte réel d'application, où les entreprises ne coopèrent que pour certaines activités et peuvent être en concurrence sur d'autres.

Pour pallier cet inconvénient, plusieurs travaux préconisent une approche distribuée. Parmi ces travaux, nous distinguons tout d'abord ceux fondés sur le paradigme multi-agents [TRA 99, TRE 98]. Sur la base d'une définition préalable des agents du système, des protocoles de coopération verticale ou horizontale sont définis permettant d'organiser et coordonner dynamiquement la production entre agents, de façon semiautomatique. D'autres travaux, tels que ceux proposés dans [MON 01] et [TEL 03], placent davantage l'homme au centre de la coopération. Ils proposent des outils ou des méthodologies permettant tout à la fois de favoriser la coopération et d'aider aux prises de décision collective. Un autre ensemble de travaux propose une approche par contrainte de la coopération [HUG 94, ERS 96, CAM 00]. Le modèle d'organisation est un réseau de centres de décision; chaque centre est caractérisé par un ensemble de variables de décision et un ensemble de contraintes reliant ces variables. Ces ensembles évoluent dynamiquement en fonction des nouvelles décisions ou des remises en cause des décisions déjà existantes. La coopération est décomposée en trois fonctions : négocier, coordonner et renégocier. Des mécanismes de propagation sous contraintes sont utilisés pour maintenir la cohérence des décisions au fur et à mesure de l'avancée du processus de coopération. Cet article s'inscrit dans la continuité de ces travaux.

\subsection{Hypothèses de travail}

Ce travail s'intéresse aux situations de coopération concernant plusieurs entreprises d'une chaîne logistique impliquées dans un processus de production à la commande. La coopération est ici envisagée comme un processus de décision distribuée. Deux types distincts de relations de coopération interentreprises peuvent être mis en évidence. Dans la relation de type donneur d'ordre-fournisseur, le but de la coopération est d'organiser la circulation du flux de produits entre les deux partenaires en minimisant les stocks, d'une part à la sortie du fournisseur et d'autre part à l'entrée de donneur d'ordre. Dans la relation de type sous-traitance, la coopération a pour but de mieux organiser la circulation du flux de produits sortant mais aussi celle flux réentrant de l'entreprise qui sous-traite. L'approche proposée dans cet article se focalise sur les relations entre donneurs d'ordre et fournisseurs. Nous supposons que les objets sur lesquels portent la coopération sont les quantités, les délais et les coûts afférents aux commandes. Dans cette optique, notre but est de définir des outils permettant de promouvoir et supporter la coopération entre une entreprise et ses partenaires.

Nous faisons l'hypothèse que chacune des fonctions de la gestion de (planification, ordonnancement, pilotage) peut être sollicitée dans une situation de coopération, ce qui amène à concevoir une architecture où chaque fonction possède son propre outil 
de coopération. En effet, le cadre temporel et organisationnel qui caractérise la relation de coopération donneur d'ordre-fournisseur diffère selon la fonction envisagée. Cet article s'intéresse principalement à la coopération concernant la fonction ordonnancement. Chaque outil de coopération étant dissocié de la fonction à laquelle il correspond, des interfaces permettant d'extraire et de formater les données détenues pas la gestion de production sont indispensables.

Comme dans [CAM 00], le mode de coopération envisagé dans cette étude est asynchrone. En effet, ce mode semble mieux adapté au contexte de la production à la commande car il ne nécessite pas une disponibilité simultanée des acteurs. Enfin, comme dans [ERS 96], nous supposons que la coopération est organisée relativement aux trois fonctions de décision : négocier, coordoner, renégocier. Nous détaillons plus précisément dans la partie suivante ce que recouvrent ces fonctions dans le cadre de la relation donneur d'ordre-fournisseur envisagée.

\section{Une approche de coopération dans les relations donneur d'ordres-fournisseurs}

\subsection{La notion de cadre de décision}

De façon classique, lorsqu'une entreprise définit les dates des approvisionnements pour la réalisation de sa production, les dates de livraisons qu'elle indique à ses fournisseurs sont antérieures aux dates auxquelles les approvisionnements sont réellement nécessaires. En effet, une entreprise préfère souvent constituer un stock d'entrée plutôt que de risquer de mettre en retard sa production à cause d'un retard d'approvisionnement. De façon symétrique, lorsqu'un fournisseur négocie des dates de livraisons avec ses clients, il se réserve lui aussi une marge de liberté afin de se conserver un peu de capacité de travail pour être capable de gérer des imprévus. Ainsi, si l'on observe globalement la tension des flux sur l'ensemble de la chaîne logistique, bien qu'elle soit localement satisfaisante, voire optimisée, elle est lâche entre chaque acteur du fait de l'existence de ces stocks tampon (cf. figure 1).

Dans le domaine de la production à la commande, l'objectif de la coopération est donc d'amener les acteurs à expliciter davantage et mieux dimensionner les marges de sécurité que chacun se réserve, pour définir les dates d'approvisionnement ou de livraison. Cette approche peut être comparée à [BAS 99] et [TSA 99] où la notion de commande contrat est utilisée dans ce but.

Pour cela, nous supposons que toute commande entre deux acteurs passe par une phase de négociation préalable dont le but est de définir un cadre de décision. Un cadre de décision représente un engagement de mise à disposition par un fournisseur d'une quantité variable de produits, dans une fenêtre temporelle, auprès de son donneur d'ordre. Ainsi, un cadre de décision peut être assimilé à un ensemble de contraintes portant sur l'état du stock de sortie d'un fournisseur dans un intervalle temporel, ou sur l'état du stock d'entrée d'un donneur d'ordre, à une translation près si les temps de transports sont pris en compte. Plusieurs cadres de décision pourront être 


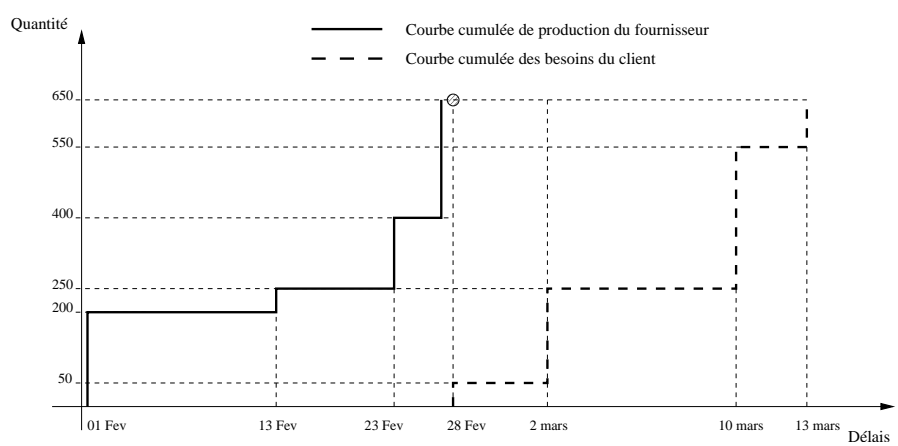

Figure 1. Courbe de production et courbe des besoins pour une même commande

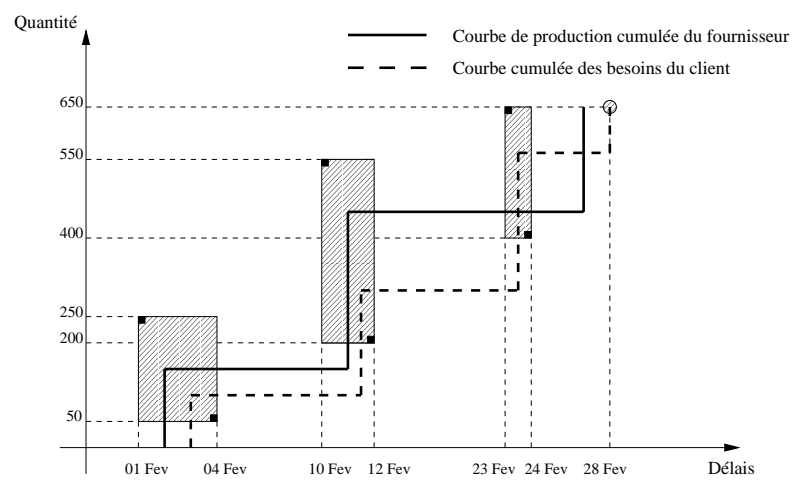

Figure 2. Cadres de décision pour une commande

associés à une même commande. La figure 2 considère par exemple une commande de 650 pièces pour le 28 février. Nous supposons que plusieurs cadres de décision, collectivement définis, prévoient de fractionner cette commande en 4 lots. Ainsi le fournisseur doit par exemple mettre à disposition du donneur d'ordre entre 200 et 550 pièces de la commande finale entre le 10 et le 12 février. Si les deux partenaires respectent ces cadres de décision, le nombre des incohérences sera diminué. Une représentation directe des cadres de décision sous forme de rectangles s'avère alors intéressante car le décideur peut disposer d'une représentation analogique directe et globale des marges de manoeuvre associées à la production. En effet, si cette représentation est enrichie par la courbe de production du fournisseur et par les quantités et les dates d'approvisionnements du donneur d'ordre, les décideurs peuvent vérifier la satisfaction des cadres de décision et détecter les incohérences. Sur la figure 2 par exemple, tous les cadres de décision sont respectés mais une incohérence concernant le troisième cadre persite. 
L'aire des rectangles et leurs positions rendent compte de la flexibilité dont dispose le fournisseur pour réaliser sa production. En effet, plus la surface d'un rectangle est importante ou, à surface égale, plus la position d'un rectangle est loin de l'origine du temps, et plus le fournisseur dispose de flexibilité pour respecter son engagement. Le bon dimensionnement d'un cadre de décision est le résultat d'un compromis qui satisfait tout à la fois les objectifs internes du fournisseur et ceux du donneur d'ordre. Un cadre de décision doit donc satisfaire deux points de vue.

Remarquons d'autre part qu'un cadre de décision n'est pas figé. Au fur et à mesure de l'avancée de la production, lorsque les besoins des clients et les plans de fabrication des fournisseurs s'affinent, les acteurs peuvent modifier les cadres de décision de sorte qu'ils soient en adéquation avec les courbes prévisionnelles de production et de demande. Ces affinements des cadres de décision se font par renégociations successives comme cela est présenté dans la partie suivante.

\subsection{Description du processus de coopération}

Un processus de négociation est initié lorsqu'un des partenaire émet une proposition : un donneur d'ordre souhaite passer une commande, ou par exemple un fournisseur veut faire une offre commerciale à un de ses donneurs d'ordre. Nous avons choisi d'illustrer ce processus de négociation à travers une IHM qui constitue une spécification pour les outils qui seront développés dans la suite de ce travail. La figure 3 illustre le cas où un fournisseur reçoit une proposition de commande concernant un produit A par le donneur d'ordre Despontin. Ce donneur d'ordre souhaite que soit mis à sa disposition entre 6 et 11 unités entre le 15 juillet $18 \mathrm{~h}$ et le 16 juillet $18 \mathrm{~h}$, pour un coût unitaire de 100 euros. Le fournisseur peut refuser ou accepter une proposition ou bien émettre une contre-proposition. De façon générale, une conversation correspond à une suite de propositions - contre-propositions et s'achève soit par une acceptation, soit par un refus.

Nous distinguons des propositions de deux natures : une proposition pour engagement qui, si elle est acceptée, induit un engagement ferme d'un fournisseur, et une proposition pour évaluation, qui induit une réponse concernant la faisabilité d'une commande (sans aucune notion d'engagement). Dans le premier cas, le processus de négociation se termine soit par un engagement (acceptation), soit par un refus. Dans le deuxième cas, le processus de négociation conclut soit à la faisabilité (acceptation), soit à l'infaisabilité (refus). Conclure à la faisabilité d'une proposition n'engage aucun des partenaires, une négociation pour engagement sera tout de même nécessaire. De plus, cette dernière n'aboutira pas nécessairement au même cadre de décision que celui déterminé lors de l'analyse de faisabilité.

La coordination consiste en l'envoi de messages. Ceux-ci peuvent être générés automatiquement et concernent les délais, les quantité ou les prix. Quatre possibilités s'offrent alors au décideur : envoyer, différer, modifier ou annuler l'envoi d'un message. 


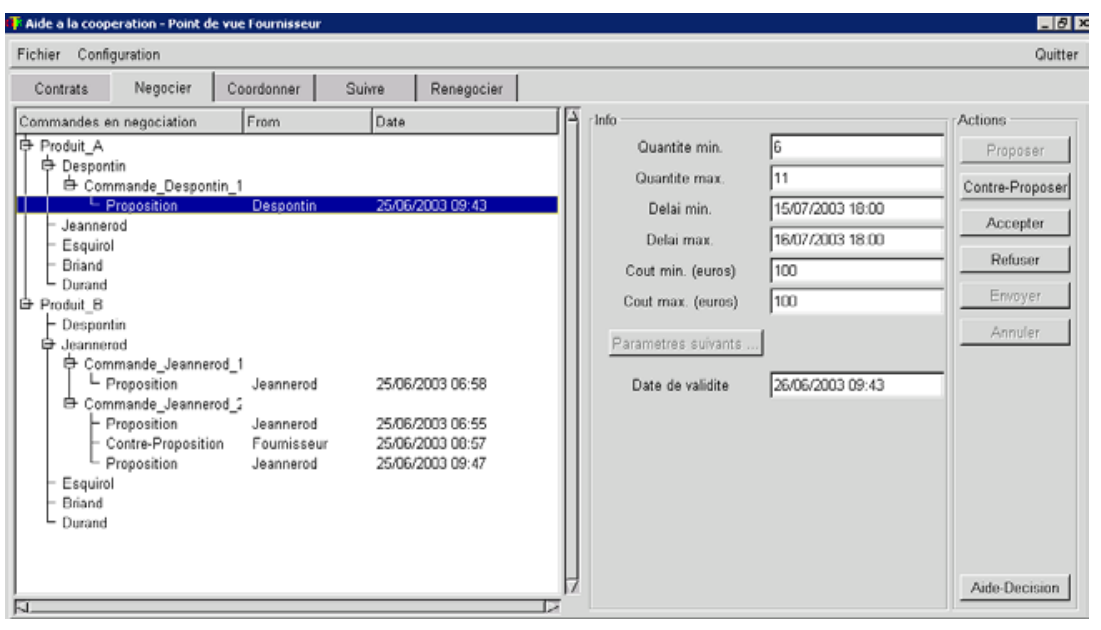

Figure 3. Représentation des conversations liées au processus de négociation

Un processus de renégociation est initié lorsqu'un des partenaires souhaite modifier un cadre de décision. Pour entamer la renégociation, le décideur émet une proposition. Le destinataire de cette demande de renégociation peut accepter ou refuser cette proposition ou bien envoyer une contre proposition.

Les contraintes sous lesquelles les processus de communication, de négociation et de renégociation peuvent se dérouler sont précisées dans les clauses d'un contrat de coopération. Ces contraintes définissent des cadres qui peuvent être déclinés selon le cadre temporel considéré, à savoir à moyen terme, à court terme ou en temps réel.

Le cadre de négociation consiste en un ensemble de contraintes qui limitent le domaine de négociation. Il peut par exemple préciser pour chaque commande les quantités minimales et maximales que l'initiateur du processus peut demander pour un cadre temporel donné, les délais minimaux et maximaux en fonction des quantités commandées, les prix minimaux et maximaux des produits en fonction des quantités commandées et des délais de livraison.

La coordination est définie par un cadre qui indique par exemple le protocole de communication utilisé par les partenaires : quand l'initiateur d'une communication envoie un message, une règle peut imposer qu'un accusé de réception lui soit retourné pour que la demande soit considérée reçue.

Le processus de négociation est lui aussi soumis à des contraintes fixées par le cadre de renégociation. Il peut préciser les variations admises en quantité, en délai et en coût entre le cadre de décision initial et le nouveau.

La définition d'un contrat de coopération constitue une tâche de nature stratégique, accomplie par chaque paire d'acteurs. Pour cela, la durée de vie d'un contrat sera 
supposée relativement longue comparée à celle d'un processus de coopération. Néanmoins, le contrat pourra être modifié pour tenir compte des variations du contexte de production.

\section{Vers un outil d'aide à la décision}

\subsection{Principe et modélisation}

Les outils de coopération doivent offrir une aide à la décision, pour la négociation et la renégociation, afin de faciliter la recherche d'un compromis satisfaisant pour les deux parties. Il s'agit de dimensionner et positionner un nouveau cadre tout en essayant de respecter les cadres de décision existants. Comme les commandes sont interdépendantes, étant réalisées sur un ensemble de ressources partagées, une fonction d'aide à la décision doit aussi montrer l'impact d'une modification d'un cadre de décision sur les autres cadres. Pour cela, ce module a besoin d'un modèle de production qui définit les ressources de l'entreprise et leur disponibilité ainsi que les gammes de production des différents produits.

À partir d'un exemple, nous allons montrer comment une approche par contrainte peut aider soit à l'insertion soit à la modification d'un cadre de décision. Par souci de simplification, nous supposons que le modèle de production est limité à une ressource de capacité unitaire, ayant un taux de production $\tau$ maximum par période de travail $T$.

La quantité de produits réalisée pour la commande $c$ au cours de la période $T$ est notée $x_{c}^{T}$. Pour chaque période $T$, les quantités minimale et maximale de pro-

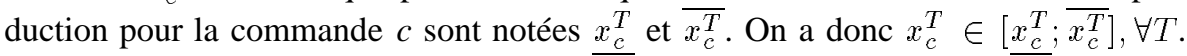
Sur $n$ périodes, la courbe de mise à disposition de la commande $c$ est notée $X_{c}^{n}=$ $\left(x_{c}^{1}, x_{c}^{2}, \ldots, x_{c}^{n}\right)$. La modélisation du problème est donnée ci-dessous.

$$
\begin{array}{lll}
\sum_{k=1}^{m} x_{k}^{T} \leq \tau & {[1]} & X_{c}^{n} \leq P_{c}^{n} \text { avec } P_{c}^{n}=\left(p_{c}^{1}, p_{c}^{2}, \ldots, p_{c}^{n}\right) \\
\sum_{k=1}^{\frac{d_{i}}{x_{0}}} x_{c}^{k} \leq \overline{q_{i}} & {[3]} & \sum_{k=1}^{\overline{d_{i}}} x_{c}^{k} \leq \overline{q_{i}} \\
\sum_{k=1}^{\overline{d_{i}}} x_{c}^{k} \geq \underline{q_{i}} & {[5]} & \sum_{k=1}^{d_{i}} x_{c}^{k} \geq \underline{q_{i}}
\end{array}
$$

Pour un nombre total de $m$ commandes dans l'atelier, l'inéquation 1 représente la contrainte de respect du taux de production de la ressource pour chaque période $T$. L'inéquation 2 modélise la contrainte imposée par la courbe de production $P_{c}^{n}$ issue de la gestion de production et concernant une commande $c$. 


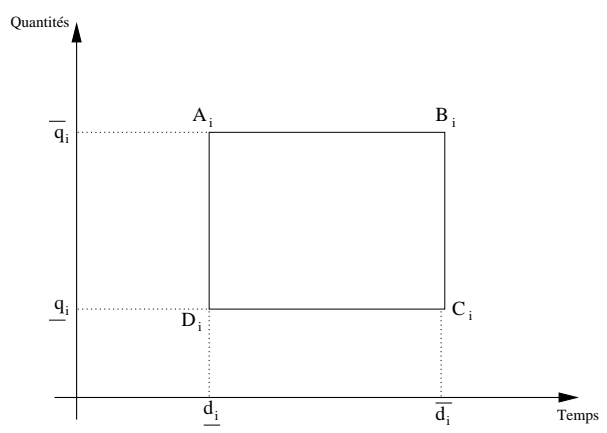

Figure 4. Cadre de décision d'une commande c

Sur la figure 4 le cadre de décision d'une commande $c$ est défini par les points $A_{i}, B_{i}, C_{i}$ et $D_{i}$. Le point $A_{i}$ indique la quantité maximale $\left(\overline{q_{i}}\right)$ qui pourra être mise à disposition au délai minimum $\left(d_{i}\right)$. Le point $B_{i}$ indique la quantité maximale qui pourra être mise à disposition au délai maximum $\left(\overline{d_{i}}\right)$. Le point $C_{i}$ indique la quantité minimale $\left(q_{i}\right)$ qui pourra être mise à disposition au délai maximum. Le point $D_{i}$ indique la quantité minimale qui pourra être mise à disposition au délai minimum. Ces contraintes sont respectivement modelisées par les inéquations 3, 4, 5 et 6 .

\subsection{Insertion d'un nouveau cadre de décision}

Pour insérer un nouveau cadre qui respecte les cadres existants, nous allons tout d'abord déterminer la position du point $A$ du nouveau cadre. Ce point représente ce que le fournisseur peut produire au maximum dans un délai minimum, c'est-à-dire lorsque les contraintes imposées par les cadres existants sont les moins contraignantes. Le point le moins contraignant pour un cadre de décision $i$ correspond au point $C_{i}$. Le point $A$ du nouveau cadre de décision est donc déterminé en prenant pour hypothèse que les courbes de mises à disposition des commandes existantes passent par le point $C$ de leur cadre de décision. En ce qui concerne les points $B$ et $D$ du nouveau cadre, ils sont déterminés sous l'hypothèse que les courbes de mise à disposition des commandes existantes respectent la courbe de production donnée par la gestion de production. En effet, ces points correspondent à la production maximum pour la nouvelle commande, lorsque la production au mieux pour les autres cadres est respectée. Enfin la position du point $C$ du nouveau cadre est déduite des positions des points $B$ et $D$ précédemment trouvées.

Illustrons cette procédure d'insertion d'un nouveau cadre de décision par un exemple. Chez un fournisseur considérons qu'il n'existe qu'une seule commande à réaliser, caractérisée par le cadre de décision 1 . Les points $A_{1}$ et $C_{1}$ correspondent respectivement aux contraintes 7 et 8 : 


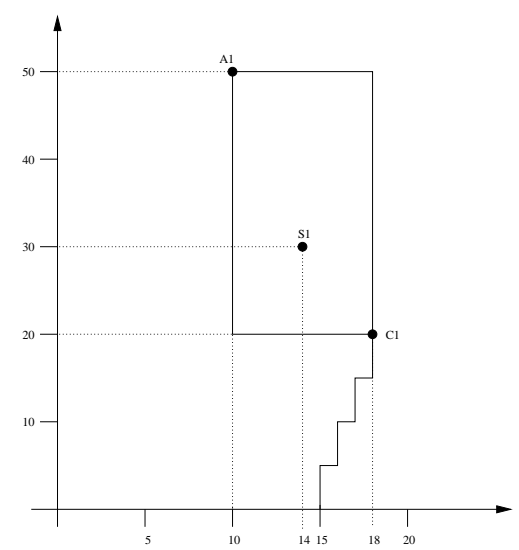

Figure 5. Cadre de décision 1

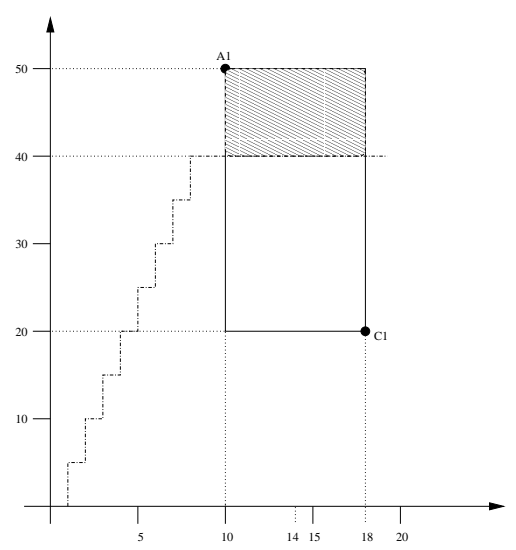

Figure 6. Impact de la modification de la deuxième commande sur la première

$$
\sum_{k=1}^{10} x_{1}^{k} \leq 50
$$

$$
\sum_{k=1}^{18} x_{1}^{k} \geq 20
$$

Le taux de production de la ressource est supposé égal à 5 unités de produits fabriqués par période $T$. Supposons également que la courbe de mise à disposition associée à cette commande soit $P_{1}^{18}=(3,3,2,2,2,2,2,2,2,2,2,2,2,2,0,0,0,0)$. L'inéquation 2 impose la contrainte : $X_{1}^{18} \leq P_{1}^{18}$. Pour la période 14 , la gestion de production a donc prévu une mise à disposition de 30 produits au maximun.

Considérons le scénario dans lequel un donneur d'ordre souhaite passer une commande et demande combien de produits le fournisseur peut lui livrer à la fin de la période 14 . Un deuxième cadre de décision doit être créé tel que $\underline{d_{2}}=14$. Il faut donc, tout d'abord, déterminer la quantité $\overline{q_{2}}=\sum_{k=1}^{14} \overline{x_{2}^{k}}$ en propageant les contraintes sur le modèle :

$$
\sum_{k=1}^{18} x_{1}^{k}=20
$$

$$
x_{1}^{T}+x_{2}^{T} \leq 5, \forall T
$$

La contrainte 9 est illustrée sur la figure 5 par la courbe de mise à disposition au plus tard passant par le point $C_{1}$. Toutes les courbes passant au dessus de cette courbe respecteront la contrainte 8 . Les contraintes 9 et 10 permettent alors de déduire le domaine de valeur de $x_{2}^{T}$ pour chaque période $T$. On obtient $\overline{q_{2}}=70$. Nous pouvons à présent déterminer la quantité $q_{2}$ et le délai $\overline{d_{2}}$ tels que $q_{2}=\sum_{k=1}^{14} \overline{x_{2}^{k}}$ et $\sum_{k=1}^{d_{2}} \overline{x_{2}^{k}}=$ $\overline{q_{2}}$. En propageant les contraintes $X_{1}^{18}=(3,3,2,2,2, \overline{2,} 2,2,2,2,2,2,2,2,0,0,0,0)$ et $x_{1}^{T}+x_{2}^{T} \leq 5$, sur le modèle, on obtient $\underline{q_{2}}=40$ et $\overline{d_{2}}=20$. 


\subsection{Modification d'un cadre de décision existant}

Si le décideur modifie un cadre de décision $i$ en le rendant moins contraignant (augmentation des délais et/ou diminution des quantités), alors la modification n'entraine aucun impact sur les autres cadres. Dans le cas contraire, en propageant les nouvelles contraintes, nous pouvons déterminer l'impact de cette modification sur les autres cadres.

Illustrons ceci en revenant sur l'exemple du paragraphe 4.2. Nous supposons un scénario dans lequel le décideur souhaite diminuer le délai maximal du cadre de décision 2 tel que $\underline{d}_{2}=18$. Par propagation de la nouvelle contrainte, le cadre de décision de la commande 2 est désormais modélisé par les inéquations 11, 12, 13 et 14 :

$$
\begin{array}{lll}
\sum_{k=1}^{14} x_{2}^{k} \leq 70 & {[11]} & \sum_{k=1}^{18} x_{2}^{k} \leq 70 \\
\sum_{k=1}^{18} x_{2}^{k} \geq 50 & {[13]} & \sum_{k=1}^{14} x_{2}^{k} \geq 50
\end{array}
$$

Les contraintes 1 et 13 imposent alors $\sum_{k=1}^{18} x_{1}^{k} \leq 40$. La zone hachurée du cadre de décision relatif à la commande 1 sur la figure 6 n'est donc plus accessible. Compte tenu de cette information, le décideur a la possibilité d'accepter la modification du cadre de décision relatif à la commande 2 ou celle de la refuser.

\section{Conclusion et perspectives}

Dans cet article, un support de coopération est proposé pour instrumenter la relation donneur d'ordre-fournisseur dans un processus de fabrication à la commande. Nous supposons que la coopération sous-tend principalement trois fonctions (négocier, coordonner et renégocier) soumises à des contraintes indiquant les règles de bon comportement en vigueur entre partenaires. Ces contraintes sont récapitulées par le contrat de coopération, cosigné par chaque paire d'acteurs décidant de former une communauté d'intérêts. Elles sont propres à chaque fonction et dépendent du cadre temporel dans lequel le processus de coopération s'inscrit. L'objet de coopération mis en jeu dans ces fonctions est le cadre de décision. Un cadre de décision est un objet partagé par donneur d'ordre et fournisseur qui définit de façon flexible les paramètres d'une commande (quantités, délais, coûts). Le cadre de décision conduit les partenaires à expliciter et définir collectivement les marges de liberté que chacun souhaite se réserver quant à la réalisation d'une commande. Le support de négociation décrit dans cet article permet de faciliter les conversations donneur d'ordre-fournisseur nécessaires à la définition d'un cadre de décision initial, et d'en maintenir une trace. Une fois un cadre de décision établi, un outil est également proposé pour supporter la coordination des partenaires tout en respectant les contraintes formalisées dans le 
contrat de coopération. Cet outil propose l'envoi semi-automatique des informations nécessaires à la coordination et permet de faciliter la détection au cours du temps des éventuelles incohérences entre productions des donneurs d'ordre et fournisseurs. Si une incohérence est mise en évidence, le cadre de décision de la commande concernée doit être renégocié. Pour cela, un support de renégociation est également proposé permettant de faciliter les conversations nécessaires à la redéfinition d'un cadre de décision cohérent.

À cause de l'interdépendance des cadres de décision des commandes, les phases de négociation et renégociation des cadres de décision doivent être assistées. Sur la base d'un modèle de production simplifié, nous avons montré comment les informations relatives aux cadres de décision et au plan de production peuvent être modélisées par un ensemble de contraintes linéaires portant sur les variables de décision mises en jeu par la coopération (quantités et délais). Ainsi, toute nouvelle décision conduit à la définition de nouvelles variables et / ou de nouvelles contraintes pouvant être propagées sur l'ensemble du modèle afin de mesurer les conséquences sur les domaines des variables déjà existantes. Cette aide à la décision permet à chaque acteur de construire à tout moment un nouveau cadre de décision totalement cohérent avec les cadres de décision déjà existants. Elle les autorise aussi à modifier un cadre de décision en mettant en évidence les répercussions de cette modificiation sur les autres cadres.

Les maquettes d'interface présentées dans cet article ont servi de base pour le développement, actuellement en cours, d'un protoype de coopération opérationnel. Ce prototype intègrerait les supports de coopération ainsi que l'aide à la décision présentés dans cet article. Le modèle de contraintes utilisé dans l'aide à la décision sera cependant plus riche, intégrant notamment l'aspect multiproduits et multiressources. La phase de développement achevée, une phase d'évaluation devra être amorcée. En effet, bien que les principes de coopération présentés dans cet article nous paraissent sains, rien ne garantit que les interfaces utilisateur et la logique d'utilisation que nous proposons soient parfaitement adaptées aux besoins des acteurs dans une situation de coopération réelle, en milieu industriel.

\section{Bibliographie}

[ART 02] Artigues C., Briand C., Portmann J.-C., Roubellat .F, « Pilotage d'atelier basé sur un ordonnancement flexible », dans « Méthodes du pilotage des systèmes de production », sous la direction de PUjo P, KIEFFER J.-P, Hermes Sciences, 2002, p. 6197.

[BAH 00] BAHROUn Z., Contribution à la planification et à la gestion dynamique des systèmes de production cyclique, Thèse de doctorat, Université de Franche-Comté, 2000.

[BAK 01] BAKER K., Greenberg S., GUTwin C., « Heuristic evaluation of groupware based on the mechanics of collaboration », Proceedings of the $8^{\text {th }}$ IFIP Working Conference on Engineering for Human-Computer Interaction, Toronto, Canada, 2001.

[BAS 99] BASSOK Y., BIXby A., SRINIVASAn R., Wiesel H.Z., « Design of componentsupply contract with commitment-revision flexibility », IBM journal of research and development, vol. 41, 1999. 
[BOU 02] Boujut J.-F., CAvaillé J.-B., Jeantet A., « Instrumentation de la coopération », dans « Coopération et connaissance dans les systèmes industriels », sous la direction de SoËnen R., Perrin J., Hermes Sciences, 2002, p. 91-109.

[BOU 01] Bourrières J.-P., Lecompte T., Deschamps J.-C., Alami R., « Un cadre formel de décision pour la planification multiniveau des systèmes de production distribués », APII-JESA, vol. 35, 2001, p. 859-883.

[CAM 00] CAMALOT J.-P., Aide à la décision et à la coopération en gestion du temps et des ressources, Thèse de doctorat, INSA de Toulouse, 2000.

[CAM 02] CAmpagne J.-P., SÉNÉChal O., « Les nouvelles exigences de coopération », dans « Coopération et connaissance dans les systèmes industriels », sous la direction de SOËNEN R., PERRIN J., Hermes Sciences, 2002, p. 51-67.

[ERS 96] ERSCHLER J., « Approche par contraintes pour l'aide à la décision et à la coopération : une nouvelle logique d'utilisation des modèles formels », dans « Coopération et conception », sous la direction de De Terssac G. , Friedberg E., Octares Editions, 1996, p. 137-147.

[GUI 00] GUINET A., «A multi-site production management approach », APII-JESA, vol. 34, 2000, p. 367-377.

[HUG 94] Huguet M.-J., Approche par contraintes pour l'aide à la décision et à la coopération en gestion de production, Thèse de doctorat, INSA de Toulouse, 1994.

[LEE 97] Lee H.L., Padmanabhan V., Whang S., « Information Distortion in a supply Chain : The Bullwhip Effect », Management science, vol. 43, 1997, p. 546-558.

[MON 01] Monteiro T., Conduite distribuée d'une coopération entre entreprises : le cas d'une relation donneurs d'ordres- fournisseurs, Thèse de doctorat, Institut National Polytechniques de Grenoble, 2001.

[SAR 02] SARdas J.-C., ERschler J., De TerssaC G., « Coopération et organisation de l'action collective », dans « Coopération et connaissance dans les systèmes industriels », sous la direction de SoËnEn R., PERrin J., Hermes Sciences, 2002, p. 69-89.

[SPO 00] SPOSITO F., «CSCW, Groupware and social issues - Applications, analytical perspectives and possible solutions in an ever-changing and critical study field », support du cours « Human-Computer Interaction », téléchargeable à l'URL http ://citeseer.nj.nec.com.

[TEL 03] TELLE O., Gestion des chaînes logistiques dans le domaine aéronautique : Aide à la coopération au sein d'une relation donneur d'ordres/fournisseurs, Thèse de doctorat, École Nationale Supérieure de l'Aéronautique et de l'Espace, 2003.

[TRA 99] Tranvouez E., Espinasse B., Ferrarini A., «Résolution coopérative et distribuée de problèmes : application multi-agents au réordonnancement d'atelier », Actes $d u$ $3^{e}$ Congrès International de Génie Industriel, l'intégration des ressources humaines et des technologies : le défi, Montréal, 1999.

[TRE 98] TRENTESAUX D., MORAY N., TAHON C., «Integration of the human operator into responsive discrete production management systems », European Journal of Operationnal Research, vol. 109, 1998, p. 342-361.

[TSA 99] TSAY A.A., LoveJOY W.S., « Quantity Flexibility Contracts and Supply Chain Performance », Manufacturing \& service operations management, vol. 1, 1999, p. 89-111. 